\title{
Myosin heavy chain isoforms in human laryngeal muscles: An expression study based on gel electrophoresis
}

\author{
LUANA TONIOLO $^{1 *}$, VERONICA MACCHI $^{1 *}$, ANDREA PORZIONATO $^{1}$, ANTONIO PAOLI $^{1}$, \\ ROSARIO MARCHESE-RAGONA ${ }^{2}$, RAFFAELE DE CARO ${ }^{1}$ and CARLO REGGIANI ${ }^{1}$ \\ ${ }^{1}$ Department of Human Anatomy and Physiology, University of Padova, Via A. Gabelli 65, I-35121 Padova; \\ ${ }^{2}$ Department of Medical and Surgical Specialities, Section of Otorhynolaryngology, \\ University of Padova, Via N. Giustiniani 2, I-35128 Padova, Italy
}

Received May 2, 2008; Accepted June 21, 2008

DOI: 10.3892/ijmm_00000033

\begin{abstract}
Laryngeal muscles in mammals are involved in highly specialized functions such as control of air passage, sphincter for airway protection and phonation. In this study, we aimed to assess whether such specialized functions are accompanied by specific distributions of fibre types. To this end, we studied the expression of the isoforms of the heavy subunit of myosin, myosin heavy chain (MyHC), which are considered the molecular markers of fibre types. The pattern of $\mathrm{MyHC}$ isoform expression was determined in 5 intrinsic laryngeal muscles: tensors as thyroarytenoid (TA), vocal (Vo), cricothyroid (CT), adductors as transverse arytenoid or interaytenoid (IA), and abductors as posterior cricoarytenoid (PCA), and 2 extrinsic muscles such as thyropharingeal $(\mathrm{TPh})$ and cricopharingeal $(\mathrm{CrPh})$. Muscles were sampled in 14 patients ( 11 males and 3 females, age 55-75 years) subjected to laryngectomy. MyHC isoforms were separated by SDS-PAGE and quantified by computer assisted densitometry. The comparison between the proportions of MyHC isoforms showed that: i) the three isoforms expressed in trunk and limb muscles (I, IIA and IIX) were present in all muscles examined, ii) the fast or type II MyHC isoforms were predominant in all muscles, representing $60-70 \%$ of the total, with no significant differences with respect to muscle types, iii) an additional isoform (indicated as MyHC L) was present in the muscles of the majority of the subjects and was more abundant in Vo and IA. MyHC L was, however, not found in any muscle of the three female patients and was never found
\end{abstract}

Correspondence to: Professor Raffaele De Caro, Section of Anatomy, Department of Human Anatomy and Physiology, University of Padova, Via A. Gabelli 65, I-35121 Padova, Italy

E-mail: rdecaro@unipd.it

${ }^{*}$ Contributed equally

Key words: human laryngeal nuscles, MHC isoforms, gel electrophoresis in TA and TPh, iv) a great inter-individual variability in MyHC distribution was present in all muscles.

\section{Introduction}

Mammalian skeletal muscles are able to respond to very different functional demands as prolonged contractile activity without fatigue to keep posture or fast and powerful contractions required, for example, for jumping or finely tuned contractions essential for speech or eye movements. Heterogeneity of muscle fibre composition represents a fundamental mechanism allowing muscles to fulfil all different functional tasks (1). Muscle fibre contractile properties are mainly determined by myosin isoform composition, kinetics of calcium release and uptake and metabolic ATP generation $(1,2)$. If compared to other mammals, the heterogeneity of human muscle fibres is limited, as type IIB and type IIM fibres are lacking and only three types, I slow, IIA and IIX fast, are present. Nevertheless, all functional requests are sufficiently covered also because the diversity among fibre types, as for example between slow and fast IIX, is greater than in other mammalian species (1).

The most accepted classification of muscle fibre types, I slow, IIA and IIX fast, is based on their myosin isoform content, which represents a major determinant of the functional heterogeneity (3). Actually, myosin is the most abundant contractile protein in muscles and myosin isoforms determine ATPase activity, maximum shortening velocity and peak power output. Myosin isoforms are thus considered the best marker for functional characterization of muscle fibres. Myosin molecule is composed of six subunits: two myosin heavy chains or MyHC and four myosin light chains or MyLC. ATPase activity and motor functions are localized in the MyHC subunits (3).

In slow fibres (type I), the MyHC I isoform is expressed and shows low enzymatic ATPase activity and generates low peak power. Generally, slow fibres have high mitochondrial content and rich blood supply, enabling them to generate ATP with aerobic oxidative metabolism. The aerobic oxidative metabolism and low ATP consumption make slow fibres fatigue resistant. Fast fibres in mammals are classified 
according to myosin content as type IIA, IIX and IIB, each expressing the corresponding MyHC isoforms. Fast fibres show high ATP consumption rate and are appropriate for short burst of high speed and power, as they lack endurance.

Mammalian muscles, however, show a repertoire of nine distinct $\mathrm{MyHC}$ isoforms characterized at protein and mRNA level (Table I). Thus, in addition to MyHC I-slow, MyHC IIA, MyHC IIX, MyHC IIB isoforms which are normally present in adult skeletal muscles, there are MHC emb and MHC neo, predominant in developing skeletal muscles, and three $\mathrm{MyHC}$ isoforms with restricted tissue distribution: MyHC $\alpha$ in masticatory muscles, MyHC eo in extraocular muscles, and MyHC $\mathrm{m}$ in masticatory muscles of carnivores. Importantly, MyHC $\alpha$ and MyHC I-slow (also indicated as $\beta$ cardiac) are major cardiac isoforms expressed in atrial and ventricular myocardium. Although most muscle fibres express only one MyHC isoform, hybrid fibres containing at the same time different MyHC isoforms are also frequent and may in some functional conditions or in some muscles represent the rule rather than the exception $(4,5)$. Different MyHCs are normally co-expressed in development, during transformation induced by electrical stimulation, and during changes in hormonal state and physical training. This observation strongly supports the idea that muscle fibre populations form a dynamic and heterogeneous system specialized to respond to different molecular and functional requests. Since isoform diversity is linked to functional diversity, the continuum of molecular phenotypes should give rise to a continuum of functional properties (3).

In those anatomical districts where muscles are not responsible for bone movements as in limbs or in chest, but control the form and the movement of organs, MyHC isoform expression can acquire very peculiar features. Eye extrinsic muscles, tongue and pharynx muscles, laryngeal intrinsic muscles, pelvic floor muscles are examples of such 'specialized' striated muscles. Their specific function might require particular and fine tuned contractions which can be generated by a unique pattern of muscle fibres and myosin isoforms. In this study, we focussed on human intrinsic and extrinsic laryngeal muscles to assess which myosin isoforms are present and how their expression fits to the unique functions of the laryngeal muscles.

Depending on their functions, human intrinsic laryngeal muscles are classified in adductors which close the glottis and include transverse arytenoid or interarytenoid (IA), and cricoarytenoid lateralis (LCA), abductors which open the glottis and include posterior cricoarytenoid (PCA), and tensors such as thyroarytenoid (TA) and cricotyroid (CT). The vocal muscle $(\mathrm{Vo})$ is a medial component of the TA and is generally considered as an tensor muscle. Semon' law stated that the abductors were subject to less voluntary control than the adductors, and therefore had less resistance to disease $(6,21)$. Abductor and adductor muscles are involved in phonation, airway protection and respiration and controversial views about their myosin isoform expression are reported in the literature (for a comprehensive review see ref. 7). The present study, based on large collection of intra-operatory samples (laryngeal muscles from 14 subjects, 11 males and 3 females) provides a complete description of the myosin isoform expression.
Table I. MyHC (myosin heavy chain) isoforms and their expression pattern.

Isoforms

\begin{tabular}{ll}
\hline MyHC 1/ß & Slow skeletal muscle fibres, heart \\
MyHC $\alpha$ & Masticatory and extraocular muscles, heart \\
MyHC IIA & Fast skeletal muscle fibres \\
MyHC IIB & Fast skeletal muscle fibres \\
MyHC IIX & Fast skeletal muscle fibres \\
MyHC emb & $\begin{array}{l}\text { Immature skeletal muscles, extraocular } \\
\text { muscles }\end{array}$ \\
MyHC neo & Immature skeletal muscles, extraocular \\
& muscles \\
MyHC eo & Extraocular muscles \\
MyHC m & Masticatory muscles (carnivores) \\
\hline
\end{tabular}

\section{Materials and methods}

Sampling of human laryngeal muscles. Laryngeal muscle samples were collected from 14 patients (11 males and 3 females, age 55-75 years) who underwent laryngectomy for neoplastic pathology. Care was taken that samples were free from neoplastic invasion. Patients were asked to give informed consent for the use of the sampled tissues for diagnostic and scientific purposes. Five intrinsic laryngeal muscles, TA, Vo, CT, IA, and PCA, and two extrinsic pharyngo-laryngeal muscles, thyropharingeal $(\mathrm{TPh})$ and cricopharyngeal $(\mathrm{CrPh})$, were sampled and immediately frozen in liquid nitrogen.

Gel electrophoresis. Muscle samples were solubilized at $90^{\circ} \mathrm{C}$ for 5 min in Laemmli buffer solution (Tris $62.5 \mathrm{mM}$, pH 6.8, glycerol 10\%, SDS 2\%, ß-mercaptoethanol 5\%, bromophenol blue $0.01 \%$ with E-64 $0.1 \%$ and leupeptin $0.1 \%$ [(Sigma, St. Louis, MO, USA) as antiproteolytic factors]. Myosin isoform expression was characterized by separating the MyHC isoforms with the following protocol. Samples were run on $8 \%$ polyacrylamide resolving gels ( $8 \%$ polyacrylamide $50: 1$, $30 \%$ glycerol, $0.4 \%$ SDS, $0.2 \mathrm{M}$ Tris, and $0.1 \mathrm{M}$ glycine) and $4 \%$ stacking gels (4\% polyacrylamide $50: 1,30 \%$ glycerol, $70 \mathrm{mM}$ Tris (pH 6.7), $4 \mathrm{mM}$ EDTA and 0.4\% SDS) after denaturation in SDS and heat with a procedure derived from Talmadge and Roy (8). Slabs $18 \mathrm{~cm}$ wide, $16 \mathrm{~cm}$ high and $1 \mathrm{~mm}$ thick were used. Electrophoresis was carried out at $4^{\circ} \mathrm{C}$ for $43 \mathrm{~h}$, at $100 \mathrm{~V}$ for the first $3 \mathrm{~h}$ and at constant $230 \mathrm{~V}$ for the remaining time. Gels were stained with Coomassie Blue (Bio-Rad) or silver stained and 4 bands were separated in $200 \mathrm{kDa}$ region (Fig. 1). MyHC isoforms were identified based on migration rate as MyHC type I, L, IIA and IIX.

For purposes of quantifying myosin isoform distribution, densitometric analyses of silver-stained bands were performed on at least two independent electrophoretic runs of each biopsy sample fragment. The mean value was used as an individual measurement. Gels were digitized with an EPSON 1650 scanner at a resolution of $1200 \mathrm{dpi}$. Each band was 


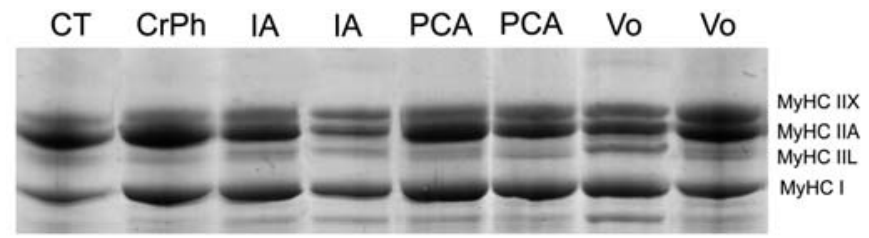

Figure 1. Example of separation of MyHC isoforms with SDS-PAGE in laryngeal muscles. A specific band migrating between MyHC I and MyHC IIA and indicated as MyHC L is visible together with the three typical bands of adult human skeletal muscles (MyHC I, MyHC IIA and MyHC IIX). In some lanes (see for example the last lane on the right) MyHC L band is split in two thin distinct bands.

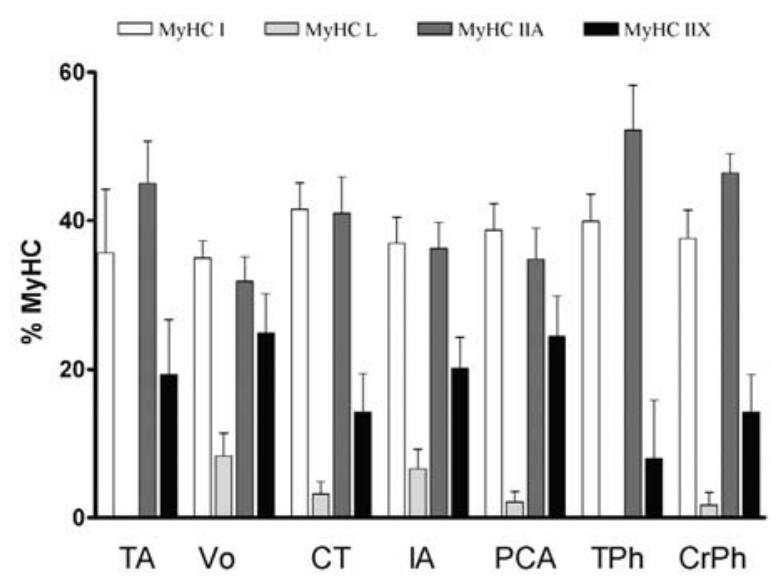

Figure 2. Distribution of $\mathrm{MyHC}$ isoforms in seven different laryngeal muscles. Results of electrophoretic separation and densitometric quantification in 14 patients are shown as means and standard errors. PCA, posterior cricoarytenoid; TA, thyroarytenoid; Vo, vocal muscle; IA, interarytenoid; CT, cricothyroid; $\mathrm{TPh}$, thyropharyngeal; $\mathrm{CrPh}$, cricopharyngeus.

characterized by a value of Brightness-Area Product (BAP) using a constant threshold after black/white inversion using Adobe Photoshop Software. In each gel, the BAP values for the bands identified as myosin heavy chain (MyHC) were summed and the BAP value for each isoform was expressed as a percent of the total. The reproducibility of the procedure was confirmed by calculating isoform ratios of selected samples from gels loaded with different amounts of sample.

Statistical analysis. Data were expressed as means and standard errors. Statistical significance of the differences between means was assessed by ANOVA followed by Student-Newman-Keuls test. A probability of $<5 \%$ was considered significant $(p<0.05)$. Statistical analysis was performed using the software PrismGraphpad.

\section{Results}

All samples of laryngeal muscles were analyzed by SDSPAGE to separate the different MyHC isoforms and quantify their expression. In the $200 \mathrm{kDa}$ region of the gels where MyHC isoforms migrate, a fourth band migrating between MyHC IIA and MyHC I was detected in many samples, in addition to the three major bands, corresponding to MyHC I, MyHC IIA and MyHC IIX respectively from the fastest migrating to the slowest. This fourth MyHC isoform is here
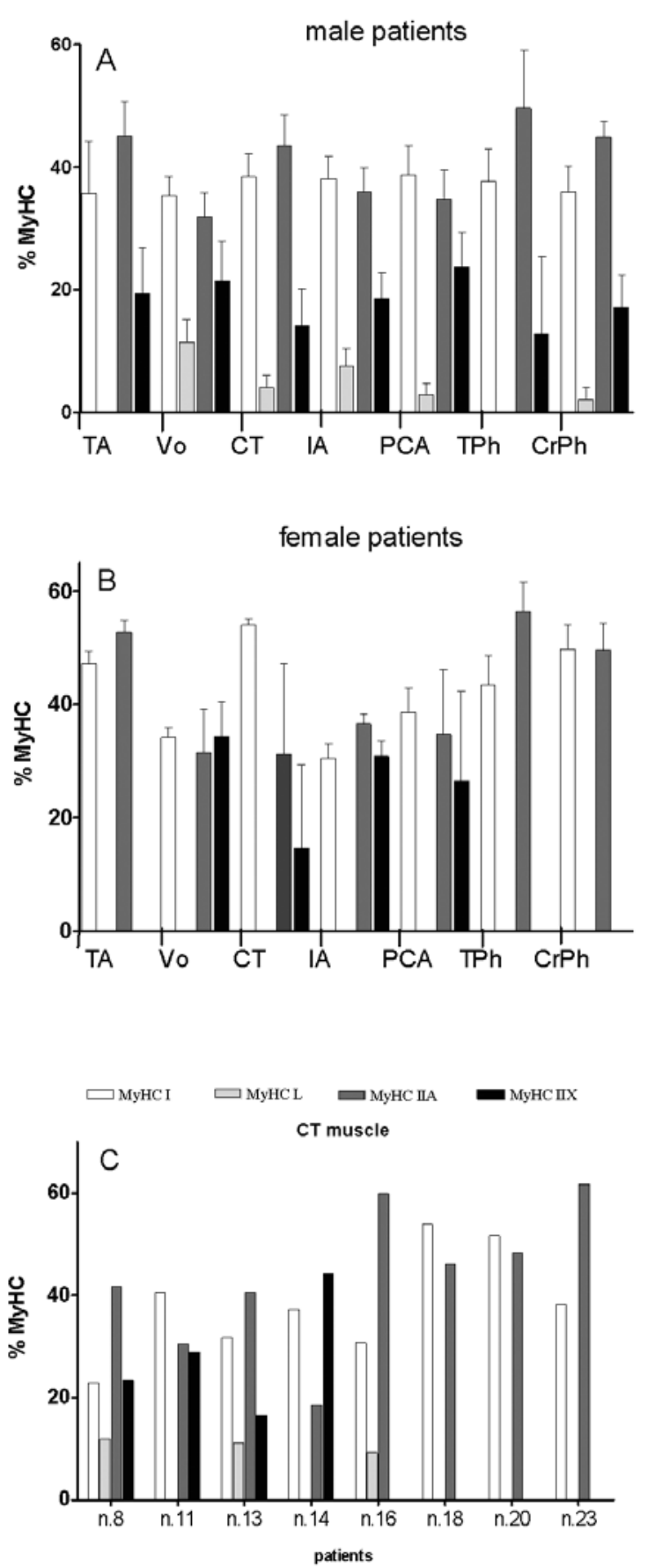

Figure 3. Distribution of MyHC isoforms in seven different laryngeal muscles. A, Average distributions in 11 male patients. B, Average distributions in 3 female patients. C, Distribution of MyHC isoforms in CT of 8 male patients. In $\mathrm{A}$ and $\mathrm{B}$ means and standard errors are shown. PCA, posterior cricoarytenoid; TA, thyroarytenoid; Vo, vocal muscle; IA, interarytenoid; CT, cricothyroid; $\mathrm{TPh}$, thyropharyngeal; $\mathrm{CrPh}$, cricopharyngeus.

referred as MyHC L (Fig. 1), as done in other studies including our own report (4), and shows a migration rate slightly faster than MyHC IIA. In some samples the fourth band was split in two minor bands (see Fig. 1, last lane on the right). MyHC $\mathrm{L}$ was present in the abductor muscle PCA, in the adductor muscle IA and in the tensors CT and Vo, but not in the TA and $\mathrm{TPh}$.

The results of the densitometric analysis are reported in Figs. 2 and 3. Fig. 2 shows the average percent distribution of MyHC isoforms of all subjects $(n=14)$ to compare expression 
of myosin isoforms in the seven muscles examined: PCA, IA, TA, Vo, CT, $\mathrm{CrPh}, \mathrm{TPh}$. As can be seen, fast isoforms are predominant in all muscles, representing together (IIA+IIX and L) $\sim 60 \%$ of the total. No significant differences are present between the proportions of slow MyHC among the seven muscles. Both MyHC IIA and MyHC IIX are expressed in all muscles, and MyHC IIA is more abundant than MyHC IIX. The specific isoform MyHC L is present in all muscles with minor amounts (the average proportion is $\leq 10 \%$ ) except $\mathrm{TA}$ and $\mathrm{TPh}$, although it is substantial in the medial part of the TA which has been considered separately and indicated as Vo.

It is important to stress that a high variability is present among the patients considered in this study. Fig. 3A and B show the average values calculated separately for the 7 muscles in the male patients $(n=11)$ and in the female patients $(n=3)$. As can be seen, in the female larynx, MyHC IIX is not present in TA, TPh and $\mathrm{CrPh}$ and $\mathrm{MyHC} \mathrm{L}$ is not expressed in any of the samples examined. Actually, the variability is great also among male patients. An example is given in Fig. 3C where the results of the densitometric analysis of the samples of CT from 8 distinct male patients are shown. MyHC IIX is lacking in 4 patients and MyHC L in 5 patients, whereas the proportion of slow o type I MyHC varies from 25 to $50 \%$.

\section{Discussion}

Craniofacial muscles are specialized to respond to special functions as ocular movement, mastication, deglutition and phonation. Such functional demands are diverse and complex and require either expression of the usual skeletal muscle MyHC isoforms (I, IIA, IIX) in particular proportions or the expression of specific MyHC isoforms normally not present in adult limb muscles. Laryngeal muscles, in particular, have three main functional tasks, as they control air passage to the lungs, they act as sphincteric device for airway protection and play an essential role in phonation. Fast closure of the glottis is needed to avoid entry of food into trachea, while fast opening is important for sneezing and coughing. Slow variations of glottis opening occur synchronously with respiration and vocal adduction and tension is required for phonation.

The results obtained in the present study show that in all laryngeal muscles sampled, fast myosin isoforms, and thus fast fibres, are more abundant than slow myosin, in an approximate ratio $2: 1$. No significant differences were found between tensor (TA, Vo, CT), adductor (IA), and abductor (PCA) muscles. Such result is at variance with that reported in some previous studies (9-11). The discrepancy can be explained taking into account the great variability in fibre types and myosin isoforms among subjects (Fig. 3). Results obtained in a small number of subjects might be misleading and, in our study, 14 patients were examined whereas only 4-5 patients were considered in other studies. For example, with reference to Fig. 3C, opposite conclusions could be obtained from patients 8, 13, 16 where MyHC I is $25-30 \%$ and from patients 18,20 where MyHC I is $50 \%$. There are, however, other possible explanations: i) the variability inside each muscle, carefully described for the vocalis of the dog by Bergrin et al (12), which might lead to different conclusions depending on the site of sampling, and ii) the possible consequences of the pathological conditions which might have caused a partial loss of muscle functions in the patients, all affected by laryngeal neoplasm. It is worth, however, to recall that both adductor and abductor muscles are asked to generate fast and slow movements as mentioned above, depending on the functional needs.

Evidence in favour of the expression of specific MyHC isoforms has been given in several studies. In rat laryngeal muscles a specific MyHC isoform (MyHC IIL) has been described by DelGaudio et al (13) and recent studies confirmed the presence of a super-fast Myosin isoform (14,15). Also in rabbit additional $\mathrm{MyHC}$ isoform has been identified in intrinsic laryngeal muscles, migrating as fast MyHC-EO isoform detected in extraocular muscles (16). In laryngeal muscles of the dog a fourth MyHC isoform has been described and identified with MyHC IIB $(12,17,18)$. The results on the presence of a fourth MyHC isoform in human laryngeal muscles are controversial, as it has been observed in some studies $(15,19,20)$ and not in other $(11,21,22)$. The electrophoretic analysis reported in the present report definitively confirms the expression of an additional myosin isoform (MyHC L) even in adult or elderly subjects and not only in infants as previously reported (20). Furthermore, our data show a remarkable variability in the expression of MyHC L which was not found in two of the seven muscles considered and was lacking in all samples collected from the female patients. Such great variability can well explain why the additional MyHC isoform has been not detected in some of the previously published studies.

While the present results strongly support the frequent expression of MyHC L, they do not provide any clue to its identification. The determination of maximum shortening velocity of hybrid fibres expressing MyHC L with MyHC IIA yielded rather low values, definitely below those obtained with pure IIA fibres (19). On this ground a possible identification with slow tonic MyHC has been forwarded by Hoh (7), but recent work has clearly demonstrated that such an isoform is not expressed in human laryngeal muscles (23). The observation of a very high shortening velocity reported in laryngeal fibres by Sciote et al (24) suggests that MyHC L might correspond to a very fast isoform such as $\mathrm{MyHC}$ eo or MyHC IIB. However, a very recent study on myosin expression at messenger level has demonstrated that both MyHC eo and IIB are not expressed in human laryngeal muscles (21). Thus, the question remains open for future studies.

In conclusion, this study provides new data on the MyHC isoform distribution in human laryngeal muscles based on a number of subjects much larger than in any previous study. The data confirm the expression of the isoforms typical of limb and trunk muscles (I, IIA, IIX) in a proportion sufficient to give a fast character to laryngeal muscles, smooth the difference in MyHC isoform proportions reported in some previous studies and demonstrate the frequent presence of a fourth MyHC isoform (MyHC L).

\section{Acknowledgements}

This study was supported by MIUR (Ministero Italiano Università Ricerca) via PRIN (Progetti di Ricerca di Interesse Nazionale) prot. N. 2005079172_002 2005. 


\section{References}

1. Bottinelli R and Reggiani C: Human skeletal muscle fibres: molecular and functional diversity. Progr Biophys Mol Biol 73: 195-262, 2000

2. Pette D and Staron RS: Cellular and molecular diversity of mammalian skeletal muscle fibers. Rev Physiol Biochem Pharmacol 116: 1-76, 1990.

3. Schiaffino S and Reggiani C: Molecular diversity of myofibrillar proteins: gene regulation and functional significance. Physiol Rev 76: 371-423, 1996.

4. Bottinelli R, Betto R, Schiaffino S and Reggiani C: Maximum shortening velocity and coexistence of myosin heavy chain isoforms in single skinned fast fibres of rat skeletal muscle. J Muscle Res Cell Motil 15: 413-419, 1994.

5. Stephenson GM: Hybrid skeletal muscle fibres: a rare or common phenomenon? Clin Exp Pharmacol Physiol 28: 692-702, 2001.

6. Vilensky JA and Sinish PR: Sir Felix Semon and Semon's law. Clin Anat 17: 605-606, 2004.

7. Hoh JF: Laryngeal muscle fibre types. Acta Physiol Scand 183: 133-149, 2005.

8. Talmadge RJ and Roy RR: Electrophoretic separation of rat skeletal muscle myosin heavy-chain isoforms. J Appl Physiol 75: 2337-2340, 1993.

9. Li ZB, Lehar M, Nakagawa H, Hoh JF and Flint PW: Differential expression of myosin heavy chain isoforms between abductor and adductor muscles in the human larynx. Otolaryngol Head Neck Surg 130: 217-222, 2004.

10. Shiotani A, Westra WH and Flint PW: Myosin heavy chain composition in human laryngeal muscles. Laryngoscope 109: 1521-1524, 1999

11. Wu YZ, Crumley RL, Armstrong WB and Caiozzo VJ: New perspectives about human laryngeal muscle: single-fiber analyses and interspecies comparisons. Arch Otolaryngol Head Neck Surg 126: 857-864, 2000.

12. Bergrin M, Bicer S, Lucas CA and Reiser PJ: Three-dimensional compartmentalization of myosin heavy chain and myosin light chain isoforms in dog thyroarytenoid muscle. Am J Physiol Cell Physiol 290: C1446-C1458, 2006.

13. DelGaudio JM, Sciote JJ, Carroll WR and Escalmado RM: Atypical myosin heavy chain in rat laryngeal muscle. Ann Otol Rhinol Laryngol 104: 237-245, 1995.
14. Shiotani A and Flint PW: Expression of extraocular-superfastmyosin heavy chain in rat laryngeal muscles. Neuroreport 9 3639-3642, 1998

15. Wu YZ, Baker MJ, Crumley RL and Caiozzo VJ: Single-fiber myosin heavy-chain isoform composition of rodent laryngeal muscle: modulation by thyroid hormone. Arch Otolaryngol Head Neck Surg 126: 874-880, 2000.

16. Lucas CA, Rughani A and Hoh JF: Expression of extraocular myosin heavy chain in rabbit laryngeal muscle. J Muscle Res Cell Motil 16: 368-378, 1995.

17. Toniolo L, Maccatrozzo L, Patruno M, Pavan E, Caliaro F, Rossi R, Rinaldi C, Canepari M, Reggiani C and Mascarello F: Fiber types in canine muscles: myosin isoform expression and functional characterization. Am J Physiol Cell Physiol 292: C1915-C1926, 2007.

18. Wu YZ, Crumley RL and Caiozzo VJ: Are hybrid fibers a common motif of canine laryngeal muscles? Single-fiber analyses of myosin heavy-chain isoform composition. Arch Otolaryngol Head Neck 126: 865-873, 2000.

19. D'Antona G, Megighian A, Bortolotto S, Pellegrino MA, Marchese-Ragona R, Staffieri A, Bottinelli R and Reggiani C: Contractile properties and myosin heavy chain isoform composition in single fibre of human laryngeal muscles. J Muscle Res Cell Motil 23: 187-195, 2002.

20. Perie S, Agbulut O, St Guily JL and Butler-Browne GS: Myosin heavy chain expression in human laryngeal muscle fibers. A biochemical study. Ann Otol Rhinol Laryngol 109: 216-220, 2000 .

21. Horton MJ, Rosen C, Close JM and Sciote JJ: Quantification of myosin heavy chain RNA in human laryngeal muscles: differential expression in the vertical and horizontal posterior cricoarytenoid and thyroarytenoid. Laryngoscope 118: 472-477, 2008.

22. Tellis CM, Rosen C, Thekdi A and Sciote JJ: Anatomy and fiber type composition of human interarytenoid muscle. Ann Otol Rhinol Laryngol 113: 97-107, 2004.

23. Sokoloff AJ, Li H and Burkholder TJ: Limited expression of slow tonic myosin heavy chain in human cranial muscles. Muscle Nerve 36: 183-189, 2007.

24. Sciote JJ, Morris TJ, Brandon CA, Horton MJ and Rosen C: Unloaded shortening velocity and myosin heavy chain variation in human laryngeal muscle fibers. Ann Otol Rhinol Laryngol 111: $120-127,2002$. 